\title{
Reconocimiento, negación y exclusión de las identidades sexuales en Le Pacte d'Afia (2009) y La bastarda (2016)
}

\author{
Edzodzomo Ondo, Hubert \\ CAEMHIL, Libreville/Gabón \\ edzodzomohu@yahoo.fr
}

\section{Resumen}

En África, la cuestión de la construcción de las identidades es difícil y compleja. Esta complejidad es debida a la dominación de la tradición que solo admite la heterosexualidad como única alternativa viable para construir la humanidad. En las sociedades como la gabonesa y la guineoecuatoriana donde hay homosexuales, lesbianas, trans, en una palabra, minoridades sexuales, cómo son tratados es la problemática que este estudio se propone aclarar. A partir de una perspectiva literaria Le pacte d'Afia, del escritor gabonés Maurice Okoumba Nkoghe, obra maestra de la literatura africana ambientada en el universo francofóno gabonés, cuenta la larga migración fang de los Eboro a la búsqueda de la « tierra prometida » por el ancestro, Nzam. Pero cuando la encuentran, una mujer, Afia, pacta con el diablo con el fin de vengarse de los maltratos e injusticias masculinos sufridos. La bastarda de la autora guineoecuatoriana, Trifonia Melibea Obono por su lado relata la caminata de Okomo, una adolescente de etnia fang que va buscando su identidad. La elección del corpus permite inscribir esta temática en la literatura fronteriza, Gabón-Guinea Ecuatorial por un lado y desde la perspectiva de los estudios del género, por otro.

\section{Abstract:}

In Africa, the question of the construction of identity is difficult and complicated. This complexity is caused by the domination of the tradition that allows only the heterosexuality as the only practicable option to construct the humanity. In the societies like the Gabonese and Guinean were gays, lesbian, transsexuals, in one word, sexual minorities, how address is the set of problems that this study intends to clarify. From a literary perspective, Le pacte d'Afia of Gabonese author Maurice Okoumba Nkoghe, greatest novel of the African literature based in the Gabonese francophone universe, relates the long migration fang of the Eboro's people to the pursuit of the "Promised Land » by the ancestor, Nzam. But, when they encounter her, a woman, Afia pacts with the Devil in order to take one's revenge on the abuse and males unfairness suffered. La bastarda of Guinean author, Trifonia Melibea as for him, reported the fair distance of Okomo, one teenager of fang ethnic group to the search of his identity. The choice of the corpus allows writing this thematic in the cross-border literature, Gabon-Equatorial Guinea on the one hand and since the perspective of the gender's studies, on the otherhand.

Palabras clave: Reconocimiento-Negación-Identidades-Gabón-Guinea Ecuatorial.

Keys words: Recognition-Refusal-Identities- Gabon-Equatorial Guinea.

\section{INTRODUCCIÓN}

En la sociedad, hombres y mujeres mantienen relaciones de dominante y dominado. Al hombre pertenece la fuerza, la autoridad, la potencia, el poder de decidir, en cuanto a la mujer, se identifica con la dulzura, la debilidad, la belleza, la sumisión, la emoción, etc. La aparición de movimientos de contestación como el feminismo para reivindicar la igualdad entre hombres y mujeres permitió evidenciar las injusticias entre ambos.

http://doi.org/10.25145/c.educomp.2018.16.036 
El nacimiento del género como categoría de análisis permitió no solo medir las injusticias entre hombres y mujeres sino posibilitó también la afirmación de las llamadas minoridades sexuales como los homosexuales, las lesbianas y los transexuales. Pero en una sociedad donde la heterosexualidad es la norma, estas identidades son negadas. ¿Pero negar el reconocimiento no es sino negar la evidencia de la existencia de estas identidades minoritarias en las sociedades? En África donde se considera que la homosexualidad es una práctica vergonzosa de los enemigos del continente, procede de Europa (Arnalte, 2016, p. 18). El estudio de dos obras de la literatura de África central, Le pacte d'Afia del autor gabonés Maurice Okoumba Nkoghe que analiza la homosexualidad como un acto diabólico, y La bastarda de la guineoecuatoriana Trifonia Melibea Obono que pone de manifiesto el calvario de los homosexuales y las lesbianas en un país donde su humanidad es negada. Una difícil condición que en ambas obras les obliga a vivir como animales. Para llevar a cabo este estudio, nos enfocamos en definir el género y en evidenciar unas observaciones sobre la noción de la performatividad según analiza Judith Butler.

Desarrollamos esta temática alrededor de tres ejes, primero marco teórico y conceptual. En este apartado vamos a definir los conceptos claves de este estudio. Luego vamos a presentar las obras al estudio. A continuación hablaremos de la negación de la humanidad de las minoridades sexuales en las sociedades de las novelas estudiadas antes de concluir.

\section{MARCO TEÓRICO Y CONCEPTUAL}

Las cuestiones de reconocimiento de identidad y de igualdad de las minoridades sexuales implican repasar la cuestión fundamental del género. Un término polisémico dado que se puede hablar del género humano o del género gramatical.

Como queda dicho, el género no es la mera traducción del sexo biológico, es la imposición de una diferencia requerida para fundar el matrimonio y el parentesco. Lo que significa que la diferencia de sexos no es natural:

Les systèmes de parenté reposent sur le mariage. Ils transforment donc des mâles et des femelles en 'hommes» et en 'femmes», chaque catégorie étant une moitié incomplète qui ne peut trouver la plénitude que dans l'union avec l'autre. Hommes et femmes sont, bien sûr, différents. Mais ils ne sont pas aussi différents que le jour et la nuit. (Fassin, 2004, p. 27).

Entonces la idea de que hombres y mujeres son dos categorías exclusivas procede de otro elemento que de la oposición natural que nunca ha sido demostrada. Entonces, las nociones de «mujer» y de «feminidad» no radican de una esencia o de un determinismo biológico sino de unaconstrucción social. Es la oposición ideológica entre el esencialismo del género y la construcción.

Una tendencia que se consolida a principio de 1990 en los Estados Unidos alrededor de la oposición sexo y género. La diferencia entre sexo y género es analizada como un lenguaje. Es la formulación crítica de Judith Butler. Para ella, el género es performativo. La performatividad es la parte del discurso que tiene la capacidad de producir lo que dice o nombra sea el género femenino sea el género masculino. (Duque, 2010).

Por ejemplo, cuando una comadrona declara al nacer un niño que es un varón o que es una hembra inmediatamente se activan los códigos sociales y culturales que hacen que el niño o la niña más tarde actúe como un hombre y un padre o una mujer y más tarde como una madre. Según explica Éric Fassin, Judith Butler ha retomado la idea fundamental de Gayle Rubin basada en el hecho de que las mujeres no son solo oprimidas como mujeres, son oprimidas sobre todo por el hecho de que tienen que ser mujeres o hombres según los casos:

Mon sentiment personnel est que le mouvement féministe doit rêver a bien plus encore qu'à l'élimination de l'oppression des femmes. Il doit rêver à l'élimination des sexualités obligatoires et des rôles de sexe. Le rêve qui me semble le plus attachant est celui d'une société androgyne et sans genre (mais pas sans sexe) où l'anatomie sexuelle n'aurait rien à voir avec qui l'on est, ce que l'on fait, ni avec qui on fait l'amour. (Ibid., pp. 33-34).

La oposición naturaleza/cultura y sexo/género, evoluciona hacia género/sexualidad. Retomando esta oposición el feminismo lesbiano en los 70 analizaba la opresión de las lesbianas como una 
opresión de mujeres. Sin embargo, las lesbianas son también oprimidas como desviantes o queers. Dos tendencias feministas se destacan hacia el año 1982, por un lado, un feminismo que se define principalmente por la lucha contra la pornografía y la prostitución y en su crítica de la dominación por la sexualidad reclamándose de Catharine Mackinnon. Por otro lado, un feminismo que se niega a definir la sexualidad por la violencia y que insiste en el «placer».

\section{PRESENTACIÓN DE LAS OBRAS AL ESTUDIO}

Le Pacte d'Afia es la decimotercera obra del autor gabonés, Maurice Okoumba Nkoghe. Se inspira del mito fang del Evus, el mal y de la epopeya del nvet. Publicada en 2009 por las ediciones La Maison Gabonaise du Livre, esta obra de ficción cuenta la larga migración de los Eboro, los inmortales desde Egipto hasta Fula-Akok, la tierra prometida por su divinidad, Nzam. Un espacio que van a compartir con un ser maligno y peligroso enviado a modo de castigo por la divinidad a causa de los abusos y exacciones cometidos durante sus andanzas. Cada uno vivía en su territorio hasta que un día, movido por el odio contra los hombres, Afia que acaba de ser desterrada por su esposo y por toda la comunidad de Fula-Akok, elijera domicilio en el territorio de la criatura maléfica. Al final, acaba pactando con ella para vengarse de todos los hombres por su egoismo, su orgullo, su brutalidad hacia los debiles y las mujeres en particular.

Una problemática retomada por Trifonia Melibea Obono en La bastarda. Es La bastarda una obra de unas ciento y diecisiete páginas que relata la historia apasionante de una joven guineoecuatoriana, Okomo de la etnia fang. Por ser incapaz de pagarle la dote a la familia política, su padre le abandona. Okomo es recogida por sus abuelos polígamos que la educan. Junto a ellos descubre las atrocidades de la poligamia, la unión matrimonial predominante en el país electa por Osá, su abuelo. Pero contra todo pronóstico, Okomo cambia radicalmente: no anhela el matrimonio en todas sus formas, huye de los hombres y prefiere relaciones con otras mujeres. Una desgracia, una abominación, concluirán Osá y todo el pueblo. Por no encontrar su sitio en la comunidad, Okomo se marcha a vivir con sus compañeros entre los cuales, su tío, Marcelo en el bosque.

\section{DE LA NEGACIÓN DE LA HUMANIDAD A LA EXCLUSIÓN SOCIAL}

En Le pacte d'Afia del autor gabonés, Maurice Okoumba Nkoghe como en La bastarda, de la guineoecuatoriana, Trifonia Melibea Obono, solo se autorizan relación sexuales entre personas de sexos distintos, masculino y femenino. Es decir que la norma para el grupo es la heterosexualidad cuyo objetivo es la reproducción. Así las relaciones consagradas tanto por la sociedad como por los ancestros en Fula Akok son las de Evormbi y Afia de la que va a nacer Meyo-Essa, Fong; la de Abut con Bindomo de la que va a nacer Alen (Okoumba Nkoghe, 2009, p. 80), etc., en la obra gabonesa por un lado y, la de Osá y sus esposas en la obra guineoecuatoriana, de la que nacen muchos hijos entre los cuales la madre de Okomo y Marcelo, por otro. Como es evidente notarlo, los hijos son el porvenir de la comunidad. Así, todo lo cuanto se aparta de esta política, la de la aplicación de la norma heterosexual es reprimida violentamente. Por ejemplo, en Le pacte d'Afia tras la muerte de sus esposos, Afia y Engongome negaron casarse de nuevo con otro miembro de la familia del difunto según recomienda la tradición o ley del levirato: «Depuis la mort de leurs époux, les deux veuves habitaient ensemble. Fières d'une liberté retrouvée, elles avaient refusé de se laisser prendre par un quelconque héritier. " (Ibid., p. 145) Una decisión condenada primero por los miembros de la comunidad luego por los ancestros. A partir de la descripción de Angone-Nzok, concluimos que la relación homosexual entre dos mujeres es anormal. Es asunto vedado en la comunidad. Es descrita como una relación bestial o animal. Se puede hablar de una deshumanización de Afia y de Engongome:

Ce qu'il vit sur le lit des veuves le surprit. Comme d'énormes lianes, deux énormes pythons se frottaient l'un contre l'autre. [...] Où étaient passées Afia et Engongome ? D'où sortaient ces reptiles? Pendant le court instant où Angone-Nzok avait l'arme brandie, les pythons, interrompant soudain leurs ébats, le fixèrent de leurs yeux ovoïdes. II remarqua alors quelque chose de tout à fait extraordinaire, qu'aucun être au monde

n'avait eu à observer avant lui : lentement, sous son regard, les deux gros reptiles prirent des visages de femmes. Les traits se précisant, il reconnut les deux veuves. (Ibid., p. 145) 
Basándose en la tradición cristiana, la metamorfosis de Afia y Engongome en reptiles no es sin recordar la representación bíblica del diablo por una enorme boa o un pitón engañando a una mujer, Eva y por ende a toda la raza humana en el Jardín de Edén con una manzana.

En suma, son Afia y Engongome las responsables de esta práctica peligrosa que amenaza con la desaparición de la especie humana. En efecto, de la relación amorosa de las dos mujeres, no nació sino el placer, la pasión amorosa pero ningún hijo excepto los que tuvieron cada una antes de la muerte de sus esposos. Ambas mujeres habían arrancado el poder entre las manos de los hombres como lo prometió el extraño animal y representaban desde aquel entonces el orgullo de todas las mujeres y el símbolo de la rebeldía. Así, eliminar hasta físicamente a las dos viudas sobre las cuales recaía la responsabilidad de todas las maldiciones de la aldea era urgente para evitar que esta práctica reprimida desde muchos siglos por los ancestros entre los Eboro se propagara:

Depuis la mort de leurs époux, les deux veuves habitaient ensemble. Fières d'une liberté retrouvée, elles avaient refusé de se laisser prendre par un quelconque héritier. Ce qui leur valait une grande admiration des autres femmes, pour lesquelles elles étaient devenues des modèles ». (Idem)

En La bastarda, en cambio, la homosexualidad, el lesbianismo y la transexualidad son prácticas femeninas y masculinas. En efecto, Dina, Pilar, Linda, Okomo y su tío, Marcelo desempeñan este papel de desviantes. Otro elemento importante que cabe subrayar es que a la diferencia de Le pacte d'Afia, donde la homosexualidad de las dos mujeres es asunto de personas maduras que gozan de cierto protagonismo político y social, la novela de Trifonia Melibea Obono, pinta personajes homosexuales, transexuales, etc más jóvenes como Dina, Pilar, Linda y Okomo pero tristes, solitarios, abandonados por los suyos a causa de su orientación sexual. Para acabar, si en Le pacte d'Afia, no sabemos cómo se llama a un homosexual, en La bastarda, Trifonia Melibea Obono asegura que entre los fang dícese de un hombre con inclinaciones homosexuales que es «hombre-mujer» o fam e mina. (Obono, 2016, p. 40). Luis Melgar Valero, autor del prólogo afirma que si los homosexuales varones son llamados con este llamamiento despectivo, humillante y burlón, es porque entre los fang, no se llama a la mujer lesbiana: «No existe ninguna palabra para designar a una mujer lesbiana. La mujer lesbiana, simplemente, no existe en la mente fang.» (Ibid., p. 14). Pero si en la mente fang la mujer lesbiana no existe, es quizá porque al principio es una práctica masculina desconocida por las mujeres.

Una tesis rechazada por Joaquín Mbomio Bacheng. Para el autor guineoecuatoriano de Matinga (2013) que vive en exilio en Francia, son las mujeres responsables de esta vergonzosa práctica en la comunidad guineoecuatoriana de antaño. En su tercera obra, relata la leyenda de la reina Mbete, del reino de Ngola, hoy Angola que se hizo tristemente famosa al enamorarse de una esclava caribeña. Precisa que fueron amores de mujeres calientes. Los ancestros castigaron tal acto pecaminoso impidiendo que regresara en Ngola, su tierra natal, convirtiendo su flota en islas e islotes que vinieron a amararse al continente negro al final de la travesía. (Mbomio Bacheng, 2013, p. 22)

Entre las razones de la marginación de Marcelo, la tribu reclama que se case con una mujer y que se reproduzca según manda la tradición. Pero no quiere consentirlo:

Un ser aislado, que estaba fuera de la sociedad ya que era un hombre-mujer, o fam e mina. Por esa razón, los hombres de la tribu le acusaban en público y en privado. Un día anunciaron en la Casa de la Palabra que ya no contaban con él como miembro de la tribu debido a su aspecto poco varonil y, sobre todo, a que no consumaba los matrimonios concertados. [...] Los hombres mayores le gritaban en la Casa de la Palabra: -jNo eres un hombre! Un varón de verdad se acuesta con mujeres y se reproduce. (Obono, 2016, p. 40)

Además se niega Marcelo a fecundar a la esposa de su hermano estéril por ser contraria a la moraleja. Pero Marcelo no se opone solo a la tradición de sus ancestros perpetuada por Osá y su primera esposa Adá por ser amoral, «La tribu exige que fecunde a mi cuñada, a la esposa del hermano de tu madre. No puedo acostarme con la mujer de mi primo por muchas razones.» (Ibid., p. 43), sino también por exclusión y marginación a las minoridades sexuales. En efecto, tras haber hecho su salida del armario, Marcelo es aislado. Le acusan de traer en el pueblo todas las desgracias que se pueden imaginar. En la carta que envía a Okomo para despedirse de ella, Marcelo ejemplifica esta desgracia:

Tus abuelos, junto con toda la tribu, me han expulsado del pueblo por varios motivos: decidí no prestar mi miembro por el bien de todos y mantengo en la vivienda las cenizas de mi padre que, según ellos, han provocado lainfertilidad de las tierras y otras desgracias en el pueblo, incluida la infertilidad de tu tío. Además, la mujer que vive conmigo es prostituta y recibe las visitas de varios hombres del pueblo, entre los que se cuenta tu abuelo. (Ibid., pp.60-61). 
Ante una sociedad resueltamente a favor de la heterosexualidad como norma de vida en Ayá Esang, su pueblo, Marcelo quiere limpiar su imagen no ante una sociedad injusta e hipócrita, que ya ha dado su veredicto por anticipación sino ante Okomo, una niña, una inocente: «Eres una niña y fácilmente influenciable. Debes saber que soy inocente.» (Ibid., p. 61).

Antes de seguir analizando los documentos, la negación de Marcelo de mantener relaciones sexuales con su cuñada encaja bien en la teoría de Judith Butler y del famoso lema de Simone de Beauvoir, «No se nace mujer uno llega a serlo». Significa que el género es el producto de una construcción, defiende ahora que incluso el sexo es una construcción social según los usos que uno le asigna. Para Marcelo como para La Manuela otro personaje homosexual de una obra cumbre de la literatura hispanoamericana, El lugar sin límites del autor José Donoso, el sexo tiene muchos usos. Si para La manuela su órgano no le sirve sino para echar orinas,

-Uno... dos... tres y chaaaaaassss... Y lanzaron a la Manuela al agua. Los hombres que la miraban desde arriba, parados entre la mora y el canal, se ahogaban de risa, señalando a la figura que hacía poses y bailaba, sumida hasta la cintura en el agua, con el vestido flotando como una mancha alrededor suyo y cantando « El relicario ». [...] Uno de los hombres trató de mear a la Manuela, que pudo esquivar el arco de la orina. Don Alejo le dio un empujón, y el hombre, maldiciendo, cayó en el agua, donde se unió durante un instante a los bailes de la Manuela. Cuando por fin les dieron la mano para que ambos subieran a la orilla todos se asombraron ante la anatomía de la Manuela. -¡Qué burro...! - Mira que está bien armado...-Psstt, si éste no parece maricón. -Que no te vean las mujeres, que se van a enamorar. La Manuela, tiritando, contestó con una carcajada. -Si este aparato no me sirve nada más que para hacer pipí. (Donoso, 2005, p. 168)

para Marcelo, su miembro no le sirve para fecundar mujeres como Osá y toda la tribu lo desea, lo utiliza por un uso poco común o distinto: «le sexe est lui-même construit et peut être modifié par l'usage des plaisirs, plaisirs qui ne correspondent pas uniquement aux formes dominantes de relation hétérosexuelle et aux jeux d'un désir «naturel»» (Bigo, 2004, p.85).

La experiencia amorosa en el bosque entre Pilar, Dina, Linda y Okomo permite afirmar que el sexo sirve solo para procurar placer: «Comenzó a besarme mientras las otras dos me iban quitando la ropa con dulzura. No puede negarme por más tiempo. Me estaba gustando y por primera vez en mi vida me sentía sexualmente libre ». (Obono, 2016, p. 65).

Contra los ataques y las acusaciones, la defensa de Marcelo es insuficiente por lo que la Iglesia también se mete en este asunto con un discurso muy agresivo contra los que se ponen al margen de la norma establecida en Ayá Esang, la heterosexualidad. Desgraciadamente, la lglesia favorece el odio y el desprecio de la sociedad hacia los homosexuales y las prostitutas:

Parece que las mujeres cuentan con el consentimiento del cura, quien asegura que la amiga de tu tío trajo el pecado al pueblo. [...] El sacerdote dice que la prostitución es pecaminosa y para que no se extienda, ella debe ser expulsada de la aldea. La manera de hacerlo ha sido idea de las mujeres de la Adoración a la Virgen María. Parece que sus esposos visitaban con frecuencia a la... puta. (Obono, 2016, pp.59-60)

A la diferencia de Restituta, la amiga de Marcelo que ha sido expulsada del pueblo, Dina, la amante de Okomo asegura que con respecto a Marcelo se trataba más bien de quemarle vivo anoche en $\mathrm{s}$ casa mientras dormía: «-Con respecto a tu tío, el verdadero objetivo de la tribu consistía en quemar la vivienda con él en el interior. Por eso la gente llevó las antorchas encendidas.» (Ibid., p. 60).

Dos ideas claves se destacan, primero la solidaridad que radica de la asociación entre Marcelo, un homosexual y Restituta, una prostituta minusválida que recuerda una terminología de Didier Bigo, el encuentro de los marginados o sea "La rencontre des «minoritaires»». Se trata precisamente de una expresión que radica de un debate famoso en 1970 sobre la temática de la diferencia entre los sexos. Como lo sabemos es la sociedad y no la diferencia biológica que construye los roles sociales y los individuos tienen que conformarse según su sexo a estos estereotipos. En definitiva, este encuentro de los marginados consistía en que el feminismo ya no trata solo de cuestiones de mujeres sino de todos aquellos que padecen de la predominancia de la heterosexualidad cuyo el hombre es el motor:

Le féminisme ne concerne plus simplement les femmes, mais tous ceux qui souffrent de la prédominancede la relation hétérosexuelle dominée par l'homme. Femmes hétérosexuelles, femmes homosexuelles, hommes homosexuels doivent se mobiliser contre le «mâle hétérosexuel» de la société capitaliste occidentale, qui se pense comme le seul normal et qui veut investir la catégorie de personne humaine à son seul profit. (Bigo, 2004, p.85) 
La otra idea esencial es que los miembros de la tribu que habían planificado quemar a Marcelo vivo recuerda que en el pueblo solo los heterosexuales merecen vivir. Los homosexuales, las lesbianas, en general los desviantes son unos enfermos que hace falta aniquilar. Expone La bastarda los pensamientos criminales así como los síntomas de la enfermedad de la que padecen (Obono, 2016, pp. 84-85). Michèle Ferrand añade que esta actitud de la comunidad se explica por el hecho de que hasta mediados de 1970 la homosexualidad era considerada como una enfermedad o un crimen. (Ferrand, 2004, p.306).

En definitiva, para evitar otros ataques y otras discriminaciones, Marcelo, Dina, Pilar, Linda y Okomo deciden instalarse en el bosque. Allí es donde viven solidariamente y libremente su homosexualidad. Una técnica conocidísima por los esclavos en el Nuevo Mundo llamado, el cimarronaje. En efecto, es en la selva donde los negros reproducían en la libertad su cultura africana que era asunto vedado en la sociedad esclavista. (Navarrete, 2001)

\section{DE LA NECESIDAD DEL RECONOCIMIENTO POLÍTICO Y SOCIAL DE LAS IDENTIDADES DE GÉNERO}

Por ser homosexuales y lesbianas son deshumanizados. Son tratados tanto en la obra gabonesa como en la guineoecuatoriana como animales que viven en la selva por no encontrar su sitio entre los hombres y las mujeres. Esta deshumanización es debido a su orientación sexual hacia el placer y no la reproducción. Es esta orientación no convencional que parece legitimar la negación de la humanidad a homosexuales y lesbianas de los que se analiza en las obras. Pero como Judith Butler lo afirma, toda vida merece ser cuidada la de un heterosexual como la de homosexuales, lesbianas y transexuales.

A pesar de ser las sociedades gabonesa y guineoecuatoriana muy conservadoras, uno de los retos importantes es aceptar la existencia de homosexuales, lesbianas u otras minoridades sexuales en sociedades donde se sigue pensando que la homosexualidad es una enfermedad y que es asunto de europeos en búsqueda de exotismo. Tales pensamientos y reflexiones demuestran a las claras que en África la gente sigue negando la evidencia cuando es menester idear un tipo de sociedad en donde cohabitan pacíficamente heterosexuales, homosexuales, lesbianas, transexuales, etc.

En La bastarda, queda también evidenciada la problemática de la adopción de los niños en una pareja homosexual, «homo-parentalité». Tras dar a luz a un niño lo que permite categorizar a Pilar como bisexual puesto que enamorada tanto de Dina, Okomo, Linda por un lado como de su padre del que es el niño y de Plácido, su prometido, por otro, huyó de su matrimonio por obligación y se fue a vivir en la selva. Allí es donde Marcelo y todo el grupo de "los indecentes» como se suele llamar a los desviantes se encarga de educar al niño. Eso para decir que como una pareja heterosexual,un homosexual es capaz de dar el amor y la educación necesarios para el bienestar y el crecimiento normal al niño (Obono, 2016, p. 111).

\section{DISCUSIÓN}

La cuestión de las minoridades que nos ha tocado hablar a través de dos obras literarias importantes de África Central, Le pacte d'Afia y La bastarda, analizan la problemática de la homosexualidad de manera distinta. Para el autor gabonés de la etnia fang, la misma a la que pertenece Trifonia Melibea Obono de Guinea Ecuatorial, esta cuestión es asunto de mujeres diabólicas y malintencionadas. En su obra, esta práctica es rechazada por ser contraria a la creencia de los ancestros. Al practicar relaciones prohibidas Afia y Engongome son consideradas como las que destruyen la relación estrecha que siempre ha existido entre los ancestros, vigías de la comunidad y la comunidad misma.

A la diferencia de Okoumba Nkoghe, Trifonia Melibea Obono apunta que hombres y mujeres practican la homosexualidad. Es acaso lo que subraya en el prólogo Luis Melgar Valero cuando dice: «Que una quiera comportarse como un hombre, jeso va en contra de nuestra tradición!» (Valero,2016, p. 12). Además aquí es donde queremos evidenciar la falsedad de la afirmación según la cual la homosexualidad «Es cosa de blanquitos, de europeos, jno es africano» (Idem) como queda dicho arriba. En su introducción a La bastarda, Arturo Arnalte destruye esta concepción mostrando que muy antes de la 
llegada de los europeos en el continente africano, ya se practicaba la homosexualidad. Para acabar su investigación, evidencia unas obras que tratan de esta cuestión. Se trata de Boy-Wives and Female Husbands. Studies in African Homosexualities de Stephen O. Murray y Will Roscoe, eds., publicado en 1988. Es una antología de textos de amores de personas del mismo sexo al sur del Sáhara entre ellas los fang; The Slaves of Timbuktu (1961) ambientada en la África occidental francesa de aquel entonces. Es un libro que Maugham Robin dedica a su joven guía local, Niangde Dakar.

Al final, si La bastarda es una obra pionera en el dominio de las cuestiones de LGTBIQ' según la concepción de Carlos Duque (Duque, 2010, p. 85) en la literatura guineoecuatoriana, cabe subrayar que en la actualidad, este tema no es desconocido en otras literaturas del continente. Por ejemplo, en Zimbabue con el libro The Hairdresser of Harare («El peluquero de Harare») del novelista Tendai Huchu cuya primera edición se publicó en Zimbabue en 2010, «Narra la dificultad de un varón homosexual para vivir su afectividad en el Zimbabue del muy homófobo Robert Mugabe y la brutalidad con que se castiga impunemente a quienes lo intentan» (Arnalte, 2016, p. 22). Podemos evocar también los libros, The Quiet violence of Dreams (2001) del sudafricano, K. Sello Duiker y Lalana (2002) de la escritora malgache Michèle Rakotosan que relata la historia de amor entre un estudiante y un músico, una pasión marcada por la tragedia del sida.

\section{CONCLUSIÓN}

En resumidas cuentas, el estudio de este trabajo nos ha permitido evidenciar la vida diaria de unas minoridades sexuales: homosexuales y lesbianas en dos obras de la literatura de África Central, primero Le pacte d'Afia del gabonés Maurice Okoumba Nkoghe luego La bastarda de la guineoecuatoriana, Trifonia Melibea Obono. Aunque estas temáticas no son centrales en las obras estudiadas, cabe decir que su condición es un verdadero calvario. En efecto, para unos, son diabólicos, malsanos, para otros no son sino unos animales. Así negada su humanidad, muchos prefieren retirarse en el bosque para escapar a eventuales ataques o asesinatos. Aunque la homosexualidad es un tabú en muchas sociedades africanas, en Gabón y en Guinea Ecuatorial en particular, su existencia social y política sigue siendo asunto vedado porque siempre se considera sin razón evidente, una práctica importada de Europa.

\section{Referencias bibliográfícas}

Arnalte, A. (2016). Introducción. Viaje iniciático hacia le libertad. Dans T. M. Obono, La bastarda (pp. 17-27). Madrid: Flores Raras.

Bigo, D. (2004). Genre et relations internationales. Dans C. Bard, C. Baudelot, \& J. Mossuz-Lavau, Quand les femmes s'en mêlent. Genre et pouvoir (pp. 80-97). Paris: Editions de La Martinière.

Donoso, J. (2005). El lugar sin límites. Madrid: Cátedra, Édition Selena Millares.

Duque, C. (2010). Judith Butler y la teoría de la performatividad de género. Revista de Educación y Pensamiento, 85-95.

\footnotetext{
${ }^{1}$ Esta abreviatura o acrónimo además de explicar algunas definiciones relacionadas con la diversidad de orientación sexual y de expresión de género busca ser descriptiva e incluyente. LGTBIQ se refiere a Lesbianas (mujeres homosexuales: es decir, orientadas erótico-afectivamente hacia personas de su mismo), Gays (hombres homosexuales), Bisexuales (personas orientadas erótico-afectivamente hacia ambos sexos), Transgénero (personas que trascienden o rompen las definiciones convencionales de hombre y mujer, no solo en su aspecto físico sino también en sus actitudes, maneras y gestos, Transexuales (personas que han decidido mediante un proceso quirúrgico cambiar sus órganos sexuales externos, Transvestis (parte del grupo transgénero, que se sienten y se visten de tiempo completo siguiendo los modelos establecidos para el género opuesto y Transformistas (transvestis ocasionales, para presentaciones o espectáculos). Intersexuales (personas que biológicamente desarrollan las características físicas y genitales de ambos sexos. Queer (del inglés: raro, extraño) que no se enmarcan en ningun tipo de categoría identitaria sexual o de género y están enmarcados en un movimiento contracultural que desafía la existencia de tales categorías para definir la diversidad sexual.
} 
Fassin, E. (2004). Le genre aux États-Unis. Dans C. Bard, C. Baudelot, \& J. Mossuz-Lavau, Quand les femmes s'en mêlent (pp. 23-43). Paris: Editions de La Martinière.

Ferrand, M. (2004). l'État, les lois du sexe et le genre. Dans C. Bard, C. Baudelot, \& J. Mossuz-Lavau, Quand les femmes s'en mêlent. Genre et pouvoir (pp. 300-323). Paris: Editions de La Martinière.

Mbomio Bacheng, J. (2013). Matinga. Sangre en la selva. Barcelona: Ediciones Mey.

Navarrete, M. C. (2001). El cimarronaje, una alternativa de libertad para los esclavos negros. Historia regional, 89-98.

Obono, T. M. (2016). La bastarda. Madrid: Flores Raras.

Okoumba Nkoghe, M. (2009). Le pacte d'Afia. Libreville: La Maison Gabonaise du livre.

Valero, L. M. (2016). Prólogo. Dans T. M. Obono, La bastarda (pp. 11-14). Madrid: Flores Raras. 